\title{
Factors Affecting Surgical Delay: A Case Study of One of General Hospital at Jeddah City
}

\author{
Baragaba Amani ${ }^{1} \&$ Alsharqi Omar $^{2}$ \\ ${ }^{1}$ Bed Management Department, King Abdulaziz Medical City, Jeddah, Saudi Arabia \\ ${ }^{2}$ Health Services and Hospitals Administration Department, Faculty of Economics and Administration, King \\ Abdulaziz University, Jeddah, Saudi Arabia \\ Correspondence: Alsharqi Omar, PhD, Department of Health Services and Hospitals Administration, King \\ Abdulaziz University, Jeddah, 21589, P O Box 80200, Saudi Arabia. Tel: 96-650-466-9776. E-mail: \\ oalsharqi@kau.edu.sa
}

Received: September 4, 2017 Accepted: September 20, 2017 Online Published: November 6, 2017

doi:10.5539/gjhs.v9n12p158 URL: https://doi.org/10.5539/gjhs.v9n12p158

\begin{abstract}
This research is a descriptive analytical study investigating the factors affecting surgical delay in the surgical department at one of general (public) hospital at Jeddah city, Saudi Arabia. The research proposes and tests four independent variable factors affecting surgical delay. These factors are: clinical, administrative, hospital capabilities and care givers, while surgical delay is the dependent variable. In order to explore this issue, a quantitative method was used to collect primary data through designing a self-administered questionnaire, which was administered at the hospital. The research targeted the surgical department's doctors at the hospital, who are the decision makers with regards to surgeries in their specialties; they total 106 doctors, and because of the small number of the research population the total number was taken as the research sample. The research retrieved 91 valid questionnaires $(96.46 \%)$. Results show that the four factors are significantly important, demonstrating a positive statistical relationship between the four factors and surgical delay. This research recommends activating the clinical coordinators' position in all surgical departments in turn, to improve the communication channels between all the concerned departments and the patients in order to run out the patients' appointments and surgery booking. Moreover, it is vital to frame, develop and manage all the surgical waiting lists in all surgical departments for easy access and control.
\end{abstract}

Keywords: surgical delay, public hospital, Saudi Arabia

\section{Introduction}

The government of the Kingdom of Saudi Arabia has given high priority to the development of the health care services sector at all levels. The latest vision of the Kingdom of Saudi Arabia, which is called Vision 2030, sets out the huge support and effort through which the Kingdom of Saudi Arabia is trying to achieve its goals, including for the health care services by 2030. The health care system in the Kingdom Saudi Arabia, like many other health care systems around the world, faces a number of concerns that continually challenge its development and growth, such as a shortage of Saudi health professionals, changing patterns of disease, the lack of a national health information system, long waiting lists for surgeries, and surgical delays.

Surgical delay is considered to be one of the most upsetting problems affecting patient satisfaction, and it is considered an important, challenging issue at most of public hospitals as the length of time a patient will wait after being booked in and registered on the waiting lists until the surgery is finally done sometimes reaches more than one year.

This research is about the factors affecting and causing surgical delay. There are four important factors to be covered that cannot be neglected in regards to surgical delay. The first factor is clinical, which includes physical and psychological factors. These include high blood pressure, which makes a major contribution to strokes, heart disease and kidney failure. Also, high cholesterol, which substantially increases the risk of coronary heart disease and stroke, and is linked to diabetes and high blood pressure. In addition, obesity and being overweight shorten life expectancy and substantially increase the risk of type 2 diabetes, heart disease, some cancers, gall bladder disease and other conditions. Examination starts when the patient attends the specialized clinic, then the doctor checks the 
patient and requests certain investigations such as radiology (MRI, CT scan, ultrasound, biopsies) and laboratory tests, then back to the clinic for a final surgical decision. Then the doctor refers the patient to the pre-anaesthesia clinic, then to a specialized department, called a post-operative or pre-admission clinic, where final clearance is made so that the patient can sign consent and the doctor can explain the surgery to the patient before he or she gets the official surgical date.

The second factor is administrative, meaning here non-medical involvement, and involving a combination of different departments connected together in the process of setting up patients' pre-surgical arrangements, such as identifying their services department and the eligibility division responsible for updating patients basic information and contact numbers, and extending eligible patients' files and limited treatment files, based on the patients providing national identification information or employees renewing their expired badges and gaining treatment approval and extensions. Administration is also responsible for setting up and printing out clinic appointments for patients. The bed management department and admissions department operate as key functions of the pure administrative process, by which all of the hospital's inpatients, day surgery and emergency admissions are combined and controlled. A supporting function for control and approval is provided on a daily basis by the operating room (OR) management to the admissions office, providing surgical list schedules and managing OR time based on the specific rules of the department. There are also other, different departments, such as clinical coordinators who are responsible for categorizing, prioritizing and managing the waiting lists based on the doctors' requests and the level of surgical need for each and every patient. This is managed along with their responsibility to call the patients and coordinate their appointments, and to finalize and send the department's surgical schedules to admissions and the OR coordinators, as well as rebook cancelled cases.

The third factor is hospital capabilities, which refers to health services resources. Bed capacity is considered to be the main element of this, which plays an important role in surgical delay as the number of beds restricts the surgical list based on the availability of beds in the male, female and/or paediatrics wards. It is also sometimes based on the surgical category depending on whether the surgery is critical or elective. In addition, the number of ICU, PICU and CCU beds can cause surgical cancellations and delays. Furthermore, the amount of medical equipment, surgery instruments and supplies plays an important role in the length of any surgery. In regards to medical instruments, the need to sterilize between each procedure might affect the surgery time, and in some cases the availability of the support equipment such as surgery scopes and devices might be one of the secondary reasons behind surgical delay.

The fourth factor is care givers, who function as the core value of any operation room, starting from the professional surgeons and then to the nursing staff, support medical staff and administrative staff. The whole process and arrangement of any surgery falls on their shoulders. Moreover, the greatest concern affecting all hospital resources is the need to recruit more manpower, based on the available budget.

The current research is focused on studying these four main factors affecting surgical delay. Furthermore, there is considerable variation to the reasons of surgical delay or cancellations all over the world. And based on that continues and different researches in this field with different formulation of the same problem and question which is surgical delay or cancellation. It is a confirmation that there is a unique profile of surgical delay problem in any institute. The results do not reflect that we just need to look in one direction and ignore the others but we do need a proper investigation, a future dynamic plan and a motivated staff to bring up the solutions out of their daily challenges that might participate in helping a needed patient for the surgery and take the institute toward success.

\section{Literature Review}

Clinics performs as a filtering resource to the surgery table as the decisions are made in specialized clinics followed by the anaesthesia clinics as a final stamp of agreement with the surgeon. The anaesthesia clinics serve to provide a pre-surgery evaluation based on the medical complexity of a patient's condition, with approval secured on completion of a health-screening questionnaire. Evaluation is made through review of the patient's medical records and relevant history, examination and relevant ancillary testing, followed by risk optimization through appropriate interventions and consultations; discussion of the risks and benefits of various anesthetic options and pain management strategies of the patients; improvement of anxiety through counselling; patient and family education on topics such as fasting, medications to continue on the day of surgery, and validation of consent and documentation of advanced medical orders (Lew, Pavlinet, \& Amundsen, 2004). The initial and most severe impact strikes when the patient and his family are waiting at the clinic for the first consultation and diagnosis. Then the patient must sit in another queue to get the MRI, CT scan, biopsy or any other investigations to complete his medical record. Finally, the patient starts the real journey, onto the surgery waiting list.

Many factors may now impact the patient adversely. The patient's name might reach the top of the operation room 
list, only for his/her surgery to be cancelled simply because there is no bed available; this might even be on the night prior to the operation, or because the patient is stressed and requests to cancel the surgery, or perhaps catches the flu or gets a respiratory infection. All of these events affect the patient, as he/she may suffer psychologically and financially. Such conditions count as physiological and psychological factors. A research conducted in a teaching hospital mentioned that "The main causes for cancelling were related to the patient $(53.4 \%)$, such as unfavourable clinical conditions (hypertension and respiratory diseases among others)" (Perroca, Jericó, \& Facundin, 2007). In other scenario, the patient might become an inpatient, and be completely ready to start the surgery, with ideal fibrotics, ultrasound, sterilized equipment, materials, surgeon and supporting medical staff, but a sudden cancellation occurs due to unavailability of an ICU or PICU bed, or the late start of a previous operation on the list, resulting in the cancellation of one or two patients. Additionally, the day surgery is considered to be one of the best solutions for both hospital and patients as well, unless there is a valid reason to transfer the patient to stay in the hospital for a couple of nights.

Overall, the length of waiting times is influenced by a number of factors. These include the number of patients needing surgery, the availability of both physical and human and material resources available for all the examinations and surgery preparations. While such surgery delays or cancelations actually cost hospitals, the waste of disposable equipment opened for surgeries that never take place, and overtime pay in the form of extra working hours for surgeons and nurses for extra surgery dated in a way to control the over demanding patients. "Surgical delay or cancelation after the patient and operating room have been prepared for surgery causes definite financial loss to the institution, while cancellations at earlier times may cause less to no real financial loss. Also, some reasons for earlier cancellations are not pertinent to very late cancellations" (Chang, Chen, Chen, Poon, \& Liu, 2014). In addition, in one study, "the main reasons for operative delay were almost the same. Lack of proper planning, lack of team work and communication gap and limited availability of trained supporting staff were the common reasons of start time operative delay" (Gupta, Agrawal, D'souza, \& Soni, 2011).

Frequent operations cancellation leads to direct waste of hospital recourses. By implementing a cohesive preoperative preparation system and schedule that may and will decrease the rate of operation cancellations significantly. It is known that junior surgeons prepares operations list, and they are unfamiliar with the procedure so they select patients whom needs minimal preparations and the study of Dhafar et al., 2015 suggested that "only consultant should book the patients for surgery and it would help in reducing cancellation rate especially in those cases which are selected because of wrong indication of surgery and furthermore, presence of consultant surgeon and consultant anaesthetist during operation reduces frequency in delays". This research focused on the decision makers as were chosen as research sample (consultants, specialists, staff physicians).

Most of the studies mentioned above demonstrate the common factors and reasons affecting surgical delay and cancellation. Moreover, it concentrated on several repeated unclassified factors which are; Patients related reasons refusal of surgery or not attending, surgeon related reason, administrative, anaesthesia, lack of theatre time (shortage of operation time were late start of the list and loss of time between cases), financial and the most repeated factor was facility related.

This research differs of the previous studies in its classified factors (clinical and it includes physical and physiological factors and it influence on the surgical delay. Administrative contains all related administrative support such as scheduling system, and admission and bed management. Finally, Health services provider's capability factor which reflect the human resources, equipment and hospital bed capacity.

Finally, surgical delay puts real pressure on any branded hospital. It threatens the hospital's reputation, financial resources, manpower necessity, service quality and patient satisfaction. The hospital clinics, administration, capabilities and care givers are the basis of its actual success, which all come together to plan for good service and excellent outcomes.

\section{Research Problem}

There are many different factors that can contribute in one way or another to surgical delay and cause patients and/or their families to suffer and complain. Also, surgeons were experiencing loss of their own precious time from their scheduled OR. This research concentrated on four factors: clinical, which includes physical and physiological factors influencing surgical delay; administrative, which comprise all related administrative support elements, including the scheduling system, admissions, and bed management; factors to do with health services providers' capabilities, which refer to human resources, status of the equipment, and hospital bed capacity; and the role of care givers, from the professional surgeons and nursing staff to the support medical and administrative staff. The current research attempts to shed light on the following main question: What are the main factors affecting surgical delay at general (public) hospitals? 


\section{Research Objective}

The research objectives are:

- Identify the critical factors which affect surgical delay in the surgery department at general hospitals.

- Investigate the input of clinical factors, administrative factors, hospital capabilities and care giver factors causing surgical delay.

\section{Research Importance}

This research seeks to make an original contribution to knowledge by investigating the most important factors affecting surgical delay. This research will help to minimize surgical delays and improve hospital performance discipline by discovering the critical factors influencing surgical delay, thereby delivering a better health service within the hospital and will contribute to improving hospital performance. This research has both academic and practical value. On the academic side, it draws attention to an under-researched area of hospital management. In practical terms, hospital managers will be able to consider how to introduce some of the research findings into their actual situations. The research design and methodology that are specifically developed for the purpose of this research should assist researchers to conduct research projects in the field of health services all over the world.

\section{Research Model and Hypotheses}

Research model is presented in Figure 1.

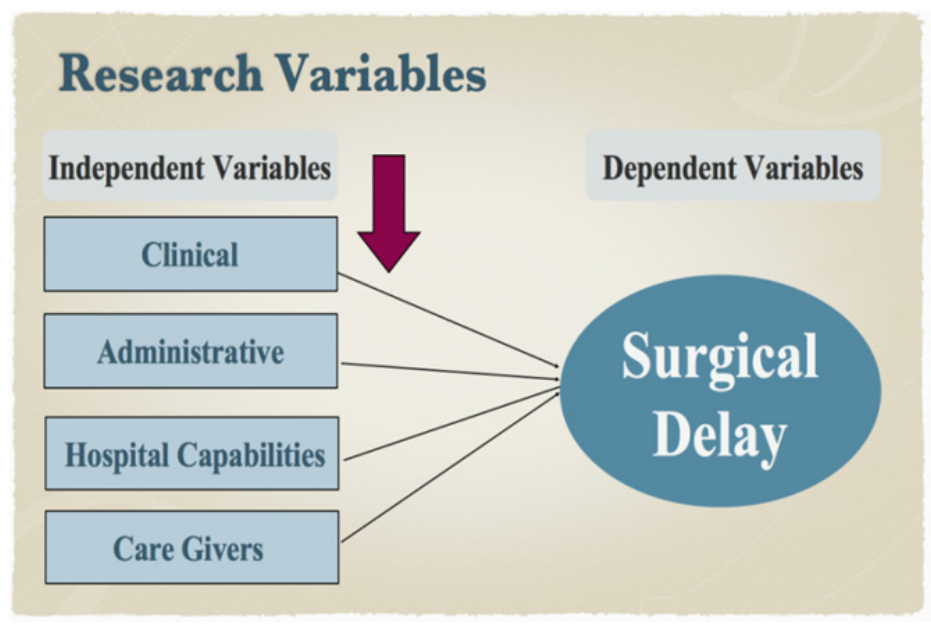

Figure 1. Research model

The research hypotheses are as the following:

- There is a significant statistical relationship between clinical factors and surgical delay.

- There is a significant statistical relationship between administrative factors and surgical delay.

- There is a significant statistical relationship between the factor of hospital capabilities and surgical delay.

- There is a significant statistical relationship between the factor of care givers and surgical delay.

\section{Research Methodology}

This study is a descriptive analytical study conducted at general (public) hospital at Jeddah city. The research tests the actual relationships that may exist between dependent and independent variables as stated in the research hypotheses. The research design constructed based on the hypotheses formulated. These hypotheses formulated inductively from the researcher's observation and from the literature review. The descriptive part needed to describe and identify the research factors, which constitute factors influencing the waiting time in the surgery department. In the analytical part, the research model being tested through examining the factors which influencing surgical delay as perceived by care givers at the hospital. Developing an effective research design is one of the fundamental parts of any research project. The research design determines research methodology, the primary data collection techniques (developing and designing the research questionnaire), research data analysis and, interpretation methods, which all affect the way the research aim and objectives are realized. Researchers classify research design into three groups: exploratory, descriptive, and analytical. This design was chosen based 
on the purpose of this study, which is to describe the state of affairs as it exists at present. The main characteristic of this method is that the researcher has no control over the variables; can only report what has happened or what is happening. Furthermore, the researchers must know and be able to delineate the parameters and what intended to measure, and find the appropriate way to measure it. There must be a clearly defined 'population' plans to study. The aim of descriptive analytical research is to obtain complete and accurate information. The design must be carefully constructed to protect against bias and achieve the utmost reliability, enabling a critical evaluation of the material. Descriptive research uses many different survey methods, including comparative and correlational methods (Kothari, 2004).

Questionnaires are most often used to collect data when the research requires knowledge about attitudes, beliefs or points of view. There are several different types, but self-administered questionnaires were chosen for their mutual advantages to both the researcher and the respondents. Self-completed questionnaires can save the researcher time and money; respondents are given more privacy and flexibility in the time required to complete them (Bryman, 2015). The research questionnaire designed on the formats of previous empirical literature and informal surgeons from different departments. The questionnaire design pre-tested and redesigned through academic staff in health services department and personal interviews with senior staff from specialist undertaking the pilot study work. The components of factors influencing the quality of patient services (manpower, resources and facilities, patients, and work system) was measured based on 5-point Likert- scale ranging from 5 (strongly agree) to 1 (strongly disagree).

\subsection{Research Population and Sampling}

It was decided that the study population for researching the surgery situation should comprise consultants, specialists and staff physicians, as final approval was gained from them. Those affected directly by surgical delay at KAMC-J were also asked to give their opinions about how and to what extent clinical and administrative factors, the hospital's capabilities, and the factor of care givers contributed to surgical delay. The population size was (106). Because of the small number of the research population, the researcher took the whole research population as the research sample. (See Table 1). The total number of the participant was 91, as there were some surgeons refused to participate and other were on annual leave and could not be reached. So the research retrieved 91 valid questionnaires $(96.46 \%)$.

Table 1. Research Population and Sample

\begin{tabular}{llll}
\hline Surgery Department & Consultant & Specialist & Staff Physician \\
\hline General Surgery & 19 & 3 & \\
Surgery Oncology & 3 & & 5 \\
Orthopedics & 17 & & 3 \\
Neurosurgery & 7 & 3 & 2 \\
Pediatric Surgery & 6 & 2 & 3 \\
Urology & 7 & & \\
ENT & 8 & 2 & 13 \\
OPhthalmology & 7 & 10 & \\
Plastic Surgery & 9 & & \\
\hline Total & 83 & & \\
& 106 & & \\
\hline
\end{tabular}

\subsection{Primary Data Collection Methods}

In this research, quantitative approaches were used for the purpose of gaining a comprehensive picture of the issues in question. Questionnaire techniques were employed to collect the primary data. The research questionnaire was designed based on previous empirical literatures. The research questionnaire was used as the primary data collection method. The components of factors influencing the quality of patient services (manpower, resources and facilities, patients, work systems) were measured on a 5-point Likert scale ranging from 5 (strongly agree) to 1 (strongly disagree). The research questionnaire was designed based on the formats provided in previous empirical literature and informally by surgeons from different departments. The questionnaire design was 
pre-tested and redesigned by academic staff in the health services department, and by personal interviews with senior staff from specialist departments at KAMC-J undertaking the pilot study work. We performed this stage following modification of some questions. The features were related to how each component affected waiting times as dependent variables.

\subsection{Research Validity and Reliability}

The research applied the reliability (Cronbach Alpha) test as follows:

Table 2. Cronbach Alpha tests

\begin{tabular}{llll}
\hline Dimension & No of items & Round 1 & Round 2 \\
\hline Patient clinical situation & 5 & 0.82 & 0.86 \\
Administrative procedures & 5 & 0.86 & 0.85 \\
Hospital capabilities & 5 & 0.82 & 0.88 \\
Care givers & 5 & 0.83 & 0.83 \\
Total & 20 & 0.88 & 0.89 \\
\hline
\end{tabular}

Table 2, Cronbach Alpha, illustrates that all constructs indicate reliability greater than the acceptable level of 0.6, indicating that the measurement errors were relatively small (Sekaran \& Bougie, 2016). The research distributed 10 questionnaires among the research respondents and gathered data from their responses. Then, data were analyzed in the first draft using Cronbach Alpha. Next, we redistributed the questionnaires among the same group and used Cronbach Alpha to analyze the data in round two, after one week. Then we distributed the research questionnaire among the whole population.

\section{Results}

\subsection{Demographic Results}

Table 3 shows the demographic results.

Table 3. Frequency and percentages for demographic variables

\begin{tabular}{llll}
\hline Variable & Demographic & Frequency & Percent \% \\
\hline \multirow{4}{*}{ Age } & Less than 30 & 0 & 0 \\
\cline { 2 - 4 } & $30-40$ & 29 & 32 \\
\cline { 2 - 4 } & $41-50$ & 33 & 37 \\
\cline { 2 - 4 } & $51-60$ & 15 & 16 \\
\cline { 2 - 4 } Total & More than 60 & 14 & 15 \\
\hline \multirow{2}{*}{ Gender } & Male & $\mathbf{9 1}$ & 90 \\
\hline Total & Female & 82 & 10 \\
\hline \multirow{2}{*}{ Nationality } & & 9 & $\mathbf{1 0 0}$ \\
\hline Total & Saudi & $\mathbf{9 1}$ & 47 \\
\hline & Non-Saudi & 43 & 53 \\
\hline \multirow{2}{*}{ Job Level } & & 48 & $\mathbf{1 0 0}$ \\
\hline Total & Consultant & $\mathbf{9 1}$ & $\mathbf{7 6}$ \\
\hline & Specialist & 69 & 11 \\
\cline { 2 - 4 } & Staff physician & 10 & 13 \\
\hline
\end{tabular}




\begin{tabular}{llll}
\hline Variable & Demographic & Frequency & Percent \% \\
\hline \multirow{3}{*}{ Experience } & Less than 5 years & 6 & 7 \\
\cline { 2 - 4 } & $5-15$ & 36 & 39 \\
\cline { 2 - 4 } & $16-25$ & 29 & 32 \\
\cline { 2 - 4 } Total & More than 25 & 20 & 22 \\
\hline
\end{tabular}

As it can be seen from table 3 the highest percentage for "age" reached (37\%) for age category (41-50) years, but the lowest percentage remained at $(0 \%)$ for the age category less than 30 years. The percentage for "gender" reached $(90 \%)$ for (male), but the percentage for (female) reached (10\%). The percentage for "nationality" reached $(53 \%)$ for (non-Saudi), but the percentage for (Saudi) reached (47\%). The percentage for "job level" reached (69\%) for (consultant), but the percentage for (specialist) reached (11\%). The highest percentage for "experience" reached (36\%) for (5-15) years, but the lowest percentage reached (7\%) and (less than 5 years). The total number of participants was 91 , as some surgeons refused to participate and others were on annual leave and could not be reached.

\subsection{Research Variables Tests (Mean, SD and Chi-Square)}

This section presents the statistical test for research variables.

1) Patient clinical situation affecting surgical delay:

Table 4 shows that the mean range was between (2.14-2.70), with the highest mean being for item (1) "Surgery complications", and the lowest mean for item (5) "Fear of moving/(wound-scar)". The overall mean for "Patient clinical situation" was (2.34). Regarding the chi-square test, the table shows that two out of five were significant.

Table 4. Mean and standard deviation for items and chi-square test/Patient's clinical situation

\begin{tabular}{llllll}
\hline No & Item & Mean & SD & Chi-square & Sig. \\
\hline 1 & Surgery complications & 2.70 & .77 & 136.23 & .01 \\
2 & Expectation of negative results & 2.55 & .81 & 119.30 & .001 \\
3 & Obesity & 2.40 & .99 & 110.92 & .12 \\
4 & Anxiety & 2.37 & .87 & 161.01 & .11 \\
5 & Fear of moving/(wound-scar) & 2.14 & .77 & 126.00 & .15 \\
& Patient clinical situation & 2.34 & & & \\
\hline
\end{tabular}

2) Administrative procedures affecting surgical delay:

Table 5 shows that mean range between (3.70-4.51), the highest mean being for item (3) "Bed management system and surgery cancellation", but the lowest mean being for item (4) "Lack of official admission clinics". The overall mean for "Administrative procedures" was (4.08). Regarding the chi-square test, the table shows that five out of five were significant.

Table 5. Mean and standard deviation for items and chi-square test/ Administrative procedures

\begin{tabular}{llllll}
\hline No & Item & Mean & SD & Chi-square & Sig. \\
\hline 1 & Long waiting list and lack of coordination & 4.33 & .67 & 226.98 & .000 \\
2 & Appointment system & 4.03 & .61 & 129.34 & .02 \\
3 & Bed management system and surgery cancellation & 4.51 & .69 & 330.91 & .01 \\
4 & Lack of official admission clinics & 3.70 & .77 & 321.01 & .002 \\
5 & Favoritism (WASTA) & 3.85 & .65 & 156.03 & .001 \\
& Administrative procedures & 4.08 & & & \\
\hline
\end{tabular}


3) Hospital capabilities affecting surgical delay:

Table 6 shows the mean range between (4.07-4.80), the highest mean being for item (1) "Availability of inpatient female beds", but the lowest mean being for item (5) "Functionality of day surgery". The overall mean for "Hospital capabilities" was (4.44). Regarding the chi-square test, the table shows that five out of five were significant.

Table 6. Mean and standard deviation for items and chi-square test/ Hospital capabilities

\begin{tabular}{llllll}
\hline No & Item & Mean & SD & Chi-square & Sig. \\
\hline 1 & Availability of inpatient female beds & 4.80 & .27 & 216.77 & .000 \\
2 & Availability of inpatient male beds & 4.59 & .64 & 219.00 & .001 \\
3 & Availability of surgery rooms & 4.37 & .59 & 170.91 & .001 \\
4 & Availability of beds in ICU, CCU , PICU and recovery & 4.40 & .89 & 221.02 & .002 \\
5 & Functionality of day surgery & 4.07 & .53 & 141.00 & .02 \\
& Hospital capabilities & 4.44 & & & \\
\hline
\end{tabular}

4) Care givers influencing surgical delay:

Table 7 shows the mean range between (2.55-4.10), the highest mean being for item (2) "Varity of surgical specialization", the lowest mean being for item (1) "Number of surgeons". The overall mean for "Care givers" was (3.41). Regarding the chi-square test, the table shows that four out of five were significant.

Table 7. Mean and standard deviation for items and chi-square test/Care givers

\begin{tabular}{llllll}
\hline No & Item & Mean & SD & Chi-square & Sig. \\
\hline 1 & Number of surgeons & 2.55 & .67 & 116.98 & .11 \\
2 & Varity of surgical specialization & 4.10 & .61 & 129.80 & .03 \\
3 & Number of nursing staff & 3.81 & .69 & 140.9 & .01 \\
4 & Number of support medical staff in OR & 3.44 & .77 & 261.01 & .002 \\
5 & Number of qualified administrative and support staff & 3.18 & .65 & 146.00 & .03 \\
& Care givers & 3.41 & & & \\
\hline
\end{tabular}

\subsection{Research Hypotheses Tests}

As it can be seen in table 8 there is a significant positive statistical relationship between clinical factors and surgical delay at KAMC-J. Therefore, the first hypothesis is accepted. There is a significant positive statistical relationship between administrative factors and surgical delay at KAMC-J. Therefore, the second hypothesis is accepted. There is a significant positive statistical relationship between the factor of hospital capabilities and surgical delay at KAMC. Therefore, the third hypothesis is accepted. Finally, there is a significant positive statistical relationship between the factor of care givers and surgical delay at KAMC-J. Therefore, the fourth hypothesis is accepted.

Table 8. Research hypotheses tests

\begin{tabular}{lll}
\hline Independent Variable & Dependent Variable: Surgical Delay & \\
\hline Clinical factors & Chi-square & Sig. \\
& 36.16 & .001 \\
Administrative factors & Chi-square & Sig. \\
& 31.15 & .003 \\
Hospital capabilities & Chi-square & Sig. \\
& 43.16 & .001 \\
Care givers & Chi-square & Sig. \\
\hline
\end{tabular}




\section{Discussion}

This section will offer a brief interpretation of the data, which have been analyzed through the system and displayed in the tables. The demographic information set out above categorizes the respondents with regard to age, gender, nationality, job level and experience. According to the results, it is clearly revealed that most (37\%) of the respondents were between (41-50) years, while the fewest percentage $(0 \%)$ occupied the age category of less than 30 years. It is also clearly revealed that most of the doctors $(90 \%)$ in the surgery department had the "gender" male, whereas the percentage for females was only $(10 \%)$. The percentage for "nationality" broke down into the majority (53\%) non-Saudi and the minority (47\%) Saudi. Regarding "job level", the percentage reached the highest (69\%) for consultant, whereas the percentage for specialist was the lowest (11\%). The percentage for "experience" was highest for 5-15 years (36\%), whereas the lowest was less than 5 years (7\%). Regarding the first dimension of the study, which is the clinical factor "patient clinical situation", the results of this general dimension was relatively low, with a rate of 2.34, and the analysis shows that the mean range was between 2.14-2.70, the highest mean being for item (1) "Surgery complications", and the lowest mean being for item (5) "Fear of moving/(wound-scar)". The results indicate that patients need proper preparation for planned surgery, as well a clear explanation of the surgery's potential complications, side effects and success rate, if possible. The patient's clinical investigations and final clarifications must also be done at the surgeon's clinic and at the anesthesia clinic for final clear-up before surgery.

Based on the previous studies mentioned earlier in chapter two, similar results were found regarding clinical reasons, but not in the percentages of the study by Dhafar, Ulmalki, Felemban, Mahfouz, Baljoon, et al. (2015), under the title "Cancellation of operations in Saudi Arabian hospitals"; the similarity was found under "Patient reasons" as a reason for surgery cancellation: patient request 59 (4.77\%); high blood pressure $51(4.12 \%)$; diabetes uncontrolled $21(1.70 \%)$; upper respiratory tract infection $14(1.13 \%)$; and ischemic heart disease $19(1.53 \%)$. Regarding the second dimension, the administrative factor "Administrative procedures", the results of this general dimension were good, with the rate of 4.08. This result shows a mean range between 3.70-4.51; the highest mean was for item (3) "Bed management system and surgery cancellation", and the lowest mean was for item (4) "Lack of official admission clinics". Regarding the chi-square test, the table shows that five out of five were significant. The bed management system monitors the patient admission flow into the hospital, as it controls the emergency, inpatients and day Surgery. Regardless, there is huge pressure from the management and the patients a continues improvements and decisions makers' meetings must be taken seriously to provide a clear guidance process or introduction of policies to the surgical department to reduce the number of regular surgery cancellations. However, there was different categorization between this research and the research conducted by El Mahalli, Al Thumairi, and Al Omar (2013), as they categorized them under "hospital reasons (procedural/ administrative)", which had the highest frequency of $57.7 \%$, followed by patients' clinical $(25.9 \%)$ and non-clinical (16.4\%) reasons. The hospital reasons (procedural/administrative) contained the category "Improper preoperative evaluation of patients", with $14.4 \%$, while in this research the lack of admission clinics serving as preoperative evaluation clinics was the lowest mean. Another item categorized under administrative by El Mahalli et al. (2013) was miscommunication, with $13.4 \%$, while in this research it was considered to be the long waiting lists and lack of coordination of communication. Another study, by Sultan et al. in (2011), conveyed this administrative factor but using a different classification to this research: No ICU beds; no blood for the patient (lack of admission clinics or preoperative clinics); and wrong bookings (long waiting list and lack of coordination). The total administrative rate in that study was $34 \%$.

Hospital capabilities are the third dimension of this research, and the results of this general dimension were the highest among all dimensions, with a rate of 4.44. The analysis shows the mean range between 4.07-4.80, with the highest mean being for item (1) "Availability of inpatient female beds", and the lowest mean being for item (5) "Functionality of day surgery". Regarding the chi-square test, the table shows that five out of five were significant. This result helps to confirm the huge need for and unavailability of enough beds, especially in the female surgical wards; generally speaking, lack of beds is the main issue actually causing surgical delay. Without doubt, there is need for continued planning, and with the new projects at KAMC-J this issue will be the main consideration of the huge expansion, and this will solve all the surgical delay problems. There were similarly high rates of cancellations revealed in the study of Dimitriadis, Iyer and Evgeniou (2013), again due to lack of beds, though they did not mention the kind of bed, i.e. if the rate was higher in male or female wards or in others. The cancelation percentage was $21.79 \%$ as a result of increased numbers of acute medical and surgical emergency admissions, whereas in this research the rate was specifically higher in the female beds, at 4.80 .

The final dimension is the care givers, and the results of this general dimension was very good with rate of 3.41. The results showed a mean range between 2.55-4.10, with the highest mean being for item (2) "Varity of surgical 
specialization", and the lowest mean being for item (1) "Number of surgeons". Regarding the chi-square test, the table shows that four out of five were significant. The results showed that the need for a variety of surgical specializations is of the highest importance; though we are not in a position to determine which kind of need this is, the result confirms how much it is relevant to surgical delay simply because most surgeons' waiting lists are full to bursting. And so, for instance, if a surgeon needs another specialized surgeon to join him in a surgery, a delay will occur based on that surgeon's busy schedule. The previous studies findings differ on this as they relay information about the huge responsibility placed on the surgeons' shoulders regarding cancellations. In one of the previous studies, by Dhafar et al. (2015), the reasons for cancelation were unavailability of surgeon with $0.24 \%$, and cancelled by surgeon with $6.95 \%$. But in the study of Sultan, Rashid, and Abbas, (2012) the related reasons for cancellation by the surgeon were listed as: the patient needs further evaluation; there is no time for surgery; no need for surgery; anticoagulants were not stopped before surgery; discharge of the patient; the surgery had already been done; illness or unavailability of the surgeon; another surgeon was asked to operate; some other reason. Additional reasons were covered in the study of Afzal and Ali (2017), who mentioned that most of the lists were being finalized by junior surgical staff, with consultants deciding only on the major cases. Junior doctors not being fully aware of the surgical plan, and their unfamiliarity with the time required for each procedure, complicated the problem.

\section{Conclusion}

In finalizing this research, and after collecting and analyzing all data, the investigation has attempted to prove or disapprove the four hypotheses, which address findings concerning the significant relation between surgical delay and clinical factors, administrative factors, and the factors of hospital capabilities and care givers. The purpose of the study was to identify and investigate the effects of the four variables on surgical delay at KAMC-J, examining and analyzing them by using an SPSS program. These factors must serve as a guide to all decision makers in solving the dilemma of surgical delay. Moreover, there is considerable variation affecting the reasons for surgical delay or cancellations all over the world. And, based on that, continuous and different researches in this field, with different formulations of the same problem, can question what causes surgical delays or cancellations. The results confirm that there is a unique profile to the problem of surgical delay in any institution. The results do not reflect that we simply need to look in one direction only and ignore all others, but we do need a proper investigation, a future dynamic plan, and a motivated staff to bring up solutions that arise from their daily challenges, solutions that might contribute to helping a patient needing surgery and, as a result, take the institute forward toward success.

In conclusion, we would like to highlight a few important points:

- A clear plan for surgery and complications must be discussed with the patient in both the pre-operative clinic and in the anesthesia clinic.

- There must be a well-developed plan from the bed management department through to the surgical department to reduce and control that continues uncontrolled admissions.

- The high demand for female surgical wards must be taken seriously, as the study shows it affects the surgical delay base.

- Some of the doctors are operating twice a month, and this leads to long waiting lists, and action must be taken either to increase the surgeons' operating days or to hire more surgeons to control the surgical delay, which sometimes reaches a year or two.

This research came up with the following recommendations based on the research results:

1) There must be a clinical coordinator in each surgical clinic in order to organize and coordinate the waiting list for each consultant.

2) There must be a clearly divided and categorized waiting list in all surgical departments, and this list should be easily accessible to all surgeons concerned so that they can be updated and aware of the long list.

3) There must be an admission or pre-operative clinic, or hours allocated in each department to allow the surgeon team to prepare patients for all the investigations they need before surgery, and this clinic should prepare a pack-up list to be available for each surgeon. Patients should be aware of it while they wait for the hospital to call.

4) The anesthesia appointment should be within a week or two to avoid any sudden infections or forgotten preparations. 


\section{Acknowledgments}

We are grateful to Dr. Alaeddin Abu-nokta for the unconditional support and for his constructive feedback and comments on the manuscript. Special Thanks to Dr. Mohammed Al-Garni for his continuous support in conducting this study.

\section{Competing Interests Statement}

All authors declare that they have no conflict of interest.

\section{References}

Afzal, F. A., \& Ali, K. A. (2017). Causes of postponement of elective surgery in Mayo Hospital Lahore. Biomedica, 26(2), 148-151.

Bryman, A. (2015). Social research methods. Oxford: Oxford University Press.

Chang, J. H., Chen, K. W., Chen, K. B., Poon, K. S., \& Liu, S. K. (2014). Case review analysis of operating room decisions to cancel surgery. BMC surgery, 14(1), 47. https://doi.org/10.1186/1471-2482-14-47

Dhafar, K. O., Ulmalki, M. A., Felemban, M. A., Mahfouz, M. E., Baljoon, M. J., Gazzaz, Z. J., .. Al-Hothali, F. T. (2015). Cancellation of operations in Saudi Arabian hospitals: Frequency, reasons and suggestions for improvements. Pakistan journal of medical sciences, 31(5), 1027. https://doi.org/10.12669/pjms.315.7932

Dimitriadis, P. A., Iyer, S., \& Evgeniou, E. (2013). The challenge of cancellations on the day of surgery. International Journal of Surgery, 11(10), 1126-1130. https://doi.org/10.1016/j.ijsu.2013.09.002

El Mahalli, A. A., Al Thumairi, A. A., \& Al Omar, R. S. (2013). On-the-Day of Surgery Cancellations of Elective Inpatient Surgeries in King Fahd Specialist Hospital in Dammam, Kingdom of Saudi Arabia. JKAU Med Sci., 20(2), 33-43. https://doi.org/10.4197/Med.20-2.4

Gupta, B., Agrawal, P., D'souza, N., \& Soni, K. D. (2011). Start time delays in operating room: Different perspectives. Saudi journal of anaesthesia, 5(3), 286-288. https://doi.org/10.4103/1658-354X.84103

Kothari, C. R. (2004). Research Methodology Methods and Techniques (2nd ed.). New Delhi: New Age International.

Lew, E., Pavlin, D. J., \& Amundsen, L. (2004). Outpatient preanaesthesia evaluation clinics. Singapore medical journal, 45(11), 509.

Perroca, M. G., Jericó, M. D. C., \& Facundin, S. D. (2007). Surgery cancelling at a teaching hospital: implications for cost management. Revista latino-americana de enfermagem, 15(5), 1018-1024. https://doi.org/10.1590/S0104-11692007000500021

Sekaran, U., \& Bougie, R. (2016). Research methods for business: A skill building approach. John Wiley \& Sons.

Sultan, N., Rashid, A., \& Abbas, S. M. (2012). Reasons for cancellation of elective cardiac surgery at Prince Sultan Cardiac Centre, Saudi Arabia. Journal of the Saudi Heart Association, 24(1), 29-34. https://doi.org/10.1016/j.jsha.2011.10.006

\section{Copyrights}

Copyright for this article is retained by the author(s), with first publication rights granted to the journal.

This is an open-access article distributed under the terms and conditions of the Creative Commons Attribution license (http://creativecommons.org/licenses/by/4.0/). 